\title{
Accident Detection using Hardware
}

\author{
Prof. J.B. Kulkarni ${ }^{1}$, Khushboo Raisinghani ${ }^{2}$, Poorva Chaturvedi ${ }^{3}$, Pranshu Sanchania ${ }^{4}$, Vartika Jharwal ${ }^{5}$ \\ Sinhgad College of Engineering, Department, Pune, India ${ }^{1,2,3,4,5}$
}

\begin{abstract}
Accident detection systems help to reduce fatalities stemming from car accident by decreasing the response time of emergency responders. The symbiosis between communication technologies and vehicles offers opportunity to improve assistance to people injured in traffic accidents, by providing information about the accident to reduce the response time of emergency assistance services. The proposed design of the application can detect accidents, pin-point the location of accident and initiate emergency communications automatically. The use of this application can significantly shorten the time taken for determination of the accident site and warning the concerned authorities. A hardware kit is used to detect the accident, get the GPS location that consists of accelerometer, Node MCU and GPS receiver. And the information about the accident location is sent to the hospitals and the relatives of the user. The message is sent through Firebase Cloud Messaging (FCM).
\end{abstract}

Keywords: Firebase cloud messaging, GPS, Node MCU.

\section{INTRODUCTION}

Today, it is very difficult to find that an accident has occurred and to find the position where it the accident occurred. It's more difficult for the lives of victims until any person know the information and informed it to the emergency vehicles like ambulance or to hospitals and if it occurs in remote areas it will becomes no hope to survive. Many efforts have been taken by the automobile manufacturers to reduce accidents. The proposed system is designed to improve the emergency assistance services, which offers detection using wireless technologies. This system does not focus on reducing number of accidents, by improving emergency assistance with efficient management of resources. The main objective of this project is to detect the vehicle accident and transmit the location of the accident with the information of victim and type of accident to hospitals and relatives. So hospitals and relatives will get the exact location by the geographical coordinates transmitted via message with the help of map.

\section{GUIDELINES FOR MANUSCRIPT PREPARATION}

\section{A. Node MCU}

NodeMCU is an open source IoT platform which includes firmware which runs on the ESP8266 Wi-Fi SoC from Espressif Systems, and hardware which is based on the ESP-12 module. NodeMCU refers to the firmware rather than the devkits. The firmware uses the Lua scripting language. It uses many open source projects, such as luacjson. NodeMCU is created after ESP2866 Came Out.

\section{B. Accelerometer}

A device that measures acceleration. They have applications in industry and science. Also used to detect and monitor vibration in rotation machinery.Used in tablet computers and digital cameras so that images on screens are always displayed upright.
Accelerometers are used in drones for flight stabilisation.

C. GPS Receiver

A GPS Receiver is a L-band radio processor capable of solving the navigation equations in order to determine the user position, velocity and precise time (PVT), by processing the signal broadcasted by GPS satellites.

\section{MATH}

The Haversine formula determines the great-circle distance between two points on a sphere given their longitudes and latitudes. Important with respect to navigation, the law of haversine, that relates the sides and angles of spherical triangles.For any two points on a sphere, the haversine of the central angle between them is given by, $\operatorname{hav}(\mathrm{d} / \mathrm{r})=\operatorname{hav}(\mathrm{O} 2-\mathrm{D})+\cos (\mathrm{O}) \cos (\mathrm{O} 2)$ hav(A2 - A)

Where,

hav is haversine function: $\operatorname{hav}(\mathrm{e})=\sin *()=1=\mathrm{Sad} d$ is the distance between the two points

ris the radius

$\mathrm{CD} 1, \mathrm{CD} 2$

: latitude of point 1 and point 2 in radians $\wedge r, \wedge$

: longitude of point 1 and point 2 in radians

\section{SYSTEM IMPLEMENTATION}

\section{A. Flowchart}

1. Waiting for accident to occur.

2. Accident is detected by accelerometer.

3. Information is sent to Node MCU

4. GPS location is extracted from GPS receiver which is given to Node MCU

5. Accident location information is given to sever and android application.

6. Server is running continuously. 
7. When coordinates are received, Haversine formula is 8 . Different lists are created according to set. The range of applied to calculate distance between Accident location hospitals can be set manually. and Hospital stored in database

\section{B. Explanation of Architecture Diagram}

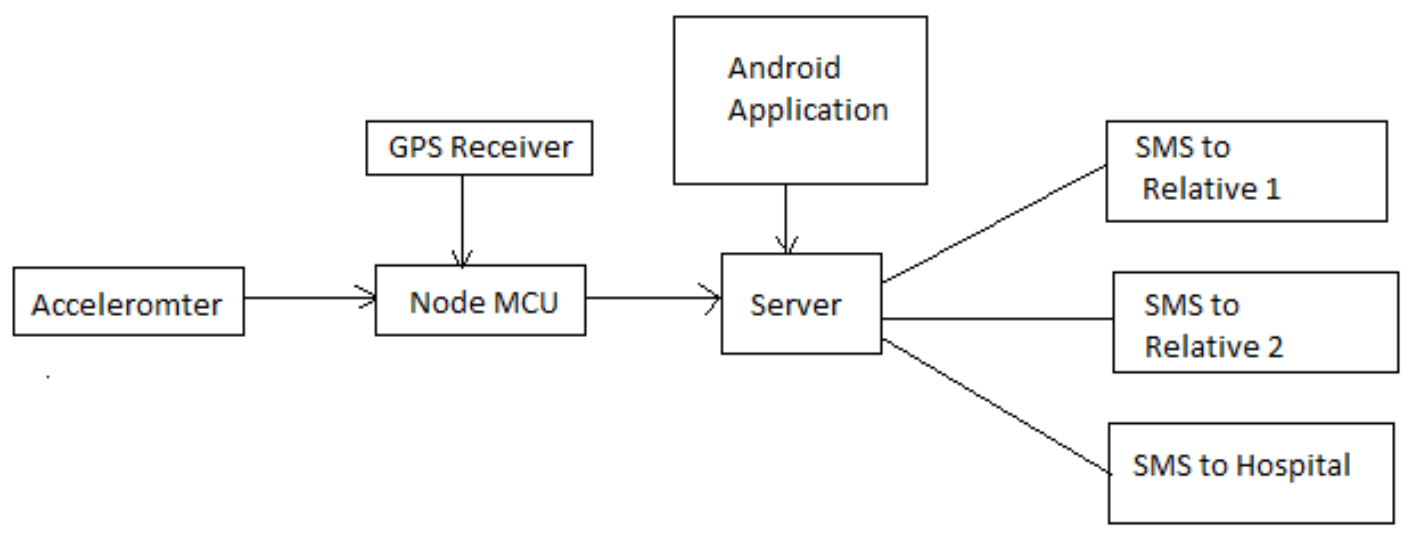

a. Accident Detection module: It consists of an accelerometer, Node MCU and GPS receiver for accident detection logic processing. The accelerometer detects change in speed of vehicle and sends the signal to Node MCU. The Node MCU is processed in such a way that if the acceleration goes above a certain limit, then it detects that the accident has occurred. The location of the accident is detected by the GPS receiver which is sent to the Node MCU. The location is detected in terms of latitude and longitude.

b. Android Application: There are two android applications for this project. First one is for the User Registration. The user registration application consists of name, mobile number, vehicle number, username, password, email id and numbers of two relatives. Second one is for the Hospital Registration which consists of name, mobile number and email id. The hospital application also consists of a Google Map that shows the accident location by the marker.

c. Database: The MYSQL database is used to store the the user registration details, the hospital registration details and also the latitudes and longitudes of accidents.

d. Server: After receiving the latitudes and longitudes of accident location, the server calculates the distance between the accident location and hospitals which are stored manually in the database by Haversine algorithm. The server will send accident location coordinates to android application, relatives and hospitals through sms. The messaging service involved here is Firebase Cloud Messaging. For calculating the distance between accident location and hospital, Haversine formula is used. The server used for processing data is Apache Tomcat server.
9. SMS is delivered to hospital and user's relatives with accident location.

\section{(1)}

A. Software Requirements:

Language: Java J2SE and JDK DE, Eclipse MARS.1

Operating System: Windows 10

Database required: MySQL

Apache Tomcat Server

Arduino 16.9

\section{B. Hardware Requirements: \\ Node MCU}

4 GB RAM

500 GB Hard disk

GPS receiver

Jumper Wires

GPS antenna

Accelerometer

\section{CONCLUSION}

The main objective of this system is to detect accidents and automate emergency assistance services. The system is sending SMS to nearest hospitals from accident location. Nearest hospital is chosen according to distance from accident location.

\section{REFERENCES}

1) Smart Vehicle Accident Detection and Alarming System. Using a Smartphone. 1st International Conference on Computer \& Information Engineering, 26-27 November, 2015.

2 Intelligent Automatic Vehicle Accident Detection System. using Wireless Communication. International Journal of Research Studies in Science, Engineering and Technology Volume 1, Issue 8, November 2014.

3) Car Accident Detection and Notification System Using Smartphone. IJCSMC, Vol. 4, Issue. 4, April 2015. 